\title{
A Switching Control Approach to Stabilization of Parameter Varying Time Delay Systems
}

\author{
Peng Yan \\ Hitay Özbay \\ Murat Şansal
}

\begin{abstract}
Robust stabilization problem is considered for time varying time delay systems, where the system parameters are scheduled along a measurable signal trajectory. A switching control approach is proposed for a class of parameter varying systems, where candidate controllers are designed for robust stabilization at certain operating regions. A dwell time based hysteresis switching logic is proposed to guarantee the stability of the switched parameter varying time delay system in the whole operating range. It is shown that if the parameter variation is slow enough (upper bound of the time derivative is determined in terms the dwell time for the switched delay system), then the system is stable with the proposed switched controllers.
\end{abstract}

\section{INTRODUCTION}

Many time varying time delay systems can be described as parameter varying systems where the system parameters are scheduled along a measurable parameter trajectory [20], [24], [27]. An example of parameter varying time delay systems is the data congestion control model for TCP networks, where all the parameters of the dynamical model, including the time delay $R T T$ (round trip time), are dependent on instantaneous queue length at the bottleneck network node [14], [28].

The analysis and control of LPV (Linear Parameter Varying) delay free systems have been discussed widely, among which two important methods are (1) gain scheduling method, and (2) switching control method. We refer to [24] for a general review on gain scheduling control methods. Additional gain scheduling design examples can be found in [1], [20]. Alternatively, the switching control method offers a new look into the design of complex control systems (e.g. nonlinear systems, parameter varying systems and uncertain systems), where the controller parameters are updated in a discrete fashion based on the switching logic. We refer to [6], [7], [11], [12], [19] and references therein for hybrid system stability analysis and switching control synthesis for systems without time delays.

There are also various recent results on LPV time delay systems [17], [21], [27]. Gain scheduling analysis and synthesis methods were investigated in [27]. In [21] stability and stabilizability were discussed for discrete time switched time delay systems; [17] considered similar stability problem in

This work is supported in part by TÜBİTAK under grant no. EEEAG$105 \mathrm{E} 156$.

P. Yan is with Enterprise Design Center, Seagate Technology LLC, 1280 Disc Drive, Shakopee, MN 55379, USA, Peng. Yan@seagate.com

H. Özbay is with Dept. of Electrical \& Electronics Engineering, Bilkent University, Bilkent, Ankara 06800, Turkey, hitay@bilkent.edu.tr

M. Şansal is with Dept. of Electrical \& Electronics Engineering, Hacettepe University, Beytepe, Ankara 06800, Turkey, sansaldee.hacettepe.edu.tr continuous time domain. Note that [21] and [17] are trajectory dependent results without taking admissible switching signals into considerations.

The present paper proposes a switching control method for robust stabilization of parameter varying time delay systems. The results of [2] are used for deriving state feedback controllers guaranteeing robust stability of the system in the neighborhoods of selected operating intervals. Then, a switching rule is developed to cover the whole operating range. More precisely, the paper derives a dwell time based stability condition for switched time varying time delay systems, which can be seen as an extension of [30]. Based on the parameter trajectory, a switching logic with hysteresis (determined by the dwell time) is proposed.

The paper is organized as follows. The switching control architecture considered for LPV time delay systems is described in Section II. In Section III, the main results on robust stabilization of LPV time delay systems are presented. The results are illustrated with a numerical example in Section IV, followed by concluding remarks in Section V.

\section{Problem Definition}

Consider the following linear parameter varying time delay systems $\Sigma_{\theta}$ for $t \geq 0$ :

$\Sigma_{\theta}:\left\{\begin{array}{c}\dot{x}(t)=A(\theta) x(t)+\bar{A}(\theta) x(t-\tau(\theta))+B(\theta) u(t) \\ x_{0}(\xi)=\phi(\xi), \quad \forall \xi \in\left[-\tau_{\max }, 0\right]\end{array}\right.$

where $x(t) \in \mathbb{R}^{n}$ is the state vector, $u(t) \in \mathbb{R}^{m}$ is control input, $\tau(\theta)$ denotes the parameter varying time-delay satisfying $0<\tau(\theta) \leq \tau_{\max }$. The LPV time delay system $\Sigma_{\theta}$ depends on a parameter $\theta(t)$, where $\theta(t) \in \mathbb{R}$ is assumed to be continuously differentiable and $\theta \in \Theta$ where $\Theta$ is a compact set.

In the present paper, we propose to construct a family of stabilizers designed at selected operating points $\theta=\theta_{i}$, $i=1,2, \ldots, l$, and perform controller switching for the above LPV time delay system, which allows for larger operating range of the LPV system. The candidate controllers are chosen from a controller set $\left\{K_{i}: i=1,2, \ldots, l\right\}$, where $K_{i}$ is a state feedback controller designed for $\theta=\theta_{i}$, which robustly stabilizes the LPV time delay systems for

$$
\theta \in \Theta_{i}:=\left[\theta_{i}^{-}, \theta_{i}^{+}\right] .
$$

An obvious necessary condition for stability of the switched system is

$$
\Theta \subseteq \bigcup_{i=1}^{l} \Theta_{i} .
$$




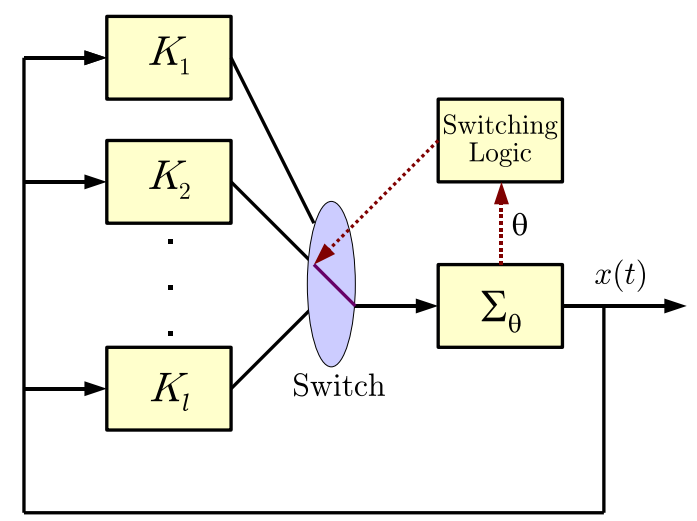

Fig. 1. The switched feedback control system

The feedback system equation can be written as:

$$
\Sigma_{q}:\left\{\begin{array}{c}
\dot{x}(t)=A_{q(t)}^{c}(\theta) x(t)+\bar{A}(\theta) x(t-\tau(\theta)), t \geq 0 \\
x_{0}(\xi)=\phi(\xi), \quad \forall \xi \in\left[-\tau_{\text {max }}, 0\right]
\end{array}\right.
$$

where $A_{q(t)}^{c}(\theta)=A(\theta)+B(\theta) K_{q(t)}$ and $q(t)$ is a piecewise switching signal taking values on the set $\mathcal{F}:=\{1,2, \ldots, l\}$, i.e. $q(t)=k_{j}, k_{j} \in \mathcal{F}$, for $\forall t \in\left[t_{j}, t_{j+1}\right)$, where $t_{j}, j \in$ $\mathbb{Z}^{+} \cup\{0\}$, is the $j^{t h}$ switching time instant which applies controller $K_{k_{j}}, u=K_{k_{j}} x$ for $\theta \in \Theta_{k_{j}}$.

In any arbitrary switching interval $t \in\left[t_{j}, t_{j+1}\right)$, we denote $\tau_{k_{j}}(\theta):=\tau(\theta)$, for $\theta \in \Theta_{k_{j}}$, and we assume

$$
\begin{aligned}
A(\theta) & =A_{k_{j}}+\Delta A(\theta), \Delta A(\theta):=D_{k_{j}} F_{k_{j}}(\theta) E_{k_{j}}, \\
\bar{A}(\theta) & =\bar{A}_{k_{j}}+\Delta \bar{A}(\theta), \Delta \bar{A}(\theta):=\bar{D}_{k_{j}} \bar{F}_{k_{j}}(\theta) \bar{E}_{k_{j}}, \\
B(\theta) & =B_{k_{j}}+\Delta B(\theta), \Delta B(\theta):=D_{k_{j}} F_{k_{j}}(\theta) E_{k_{j}}^{B}
\end{aligned}
$$

where we further assume that

$$
F_{k_{j}}(\theta)^{T} F_{k_{j}}(\theta) \leq I \quad \text { and } \quad \bar{F}_{k_{j}}(\theta)^{T} \bar{F}_{k_{j}}(\theta) \leq I
$$

It is clear that the trajectory of $\Sigma_{q}$ in any arbitrary switching interval $t \in\left[t_{j}, t_{j+1}\right)$ can be expressed:

$\Sigma_{k_{j}}:\left\{\begin{aligned} \dot{x}(t)= & \left(A_{k_{j}}^{c}+\Delta A_{k_{j}}^{c}(\theta)\right) x(t) \\ & +\left(\bar{A}_{k_{j}}+\Delta \bar{A}(\theta)\right) x\left(t-\tau_{k_{j}}(\theta)\right) \\ x_{t_{j}}(\xi)= & \phi_{j}(\xi), \quad \forall \xi \in\left[-\bar{\tau}_{k_{j}}, 0\right],\end{aligned}\right.$

where $0<\bar{\tau}_{k_{j}}:=\max \tau_{k_{j}}(\theta)$, for $\theta \in \Theta_{k_{j}}, \phi_{j}(\xi)$ is defined as:

$$
\phi_{j}(\xi)=\left\{\begin{array}{lr}
x\left(t_{j}+\xi\right) & -\bar{\tau}_{k_{j}} \leq \xi<0 \\
\lim _{h \rightarrow 0^{-}} x\left(t_{j}+h\right), & \xi=0
\end{array}\right.
$$

and

$$
\begin{aligned}
A_{k_{j}}^{c} & =A_{k_{j}}+B_{k_{j}} K_{k_{j}}, \\
\Delta A_{k_{j}}^{c} & =D_{k_{j}} F_{k_{j}}(\theta) E_{k_{j}}^{c}, \\
E_{k_{j}}^{c} & =E_{k_{j}}+E_{k_{j}}^{B} K_{k_{j}}
\end{aligned}
$$

In the following section, we will establish sufficient conditions on the stability of the switched systems (4), as well as the robust stabilization of LPV time delay systems (1).

\section{Main Results}

First we define the notation used in this section: as usual $\|\cdot\|$ denotes the Euclidean norm in $\mathbb{R}^{n}$, and for a continuous function $f \in C\left([t-r, t], \mathbb{R}^{n}\right)$ we define

$$
|f|_{[t-r, t]}:=\sup _{t-r \leq \theta \leq t}\|f(\theta)\| .
$$

As in [30], we say that the switched time-delay system $\Sigma_{q}$ described by (4) is stable if there exists a continuous strictly increasing function $\bar{\alpha}: \mathbb{R}^{+} \rightarrow \mathbb{R}^{+}$with $\bar{\alpha}(0)=0$ such that

$$
\|x(t)\| \leq \bar{\alpha}\left(|x|_{\left[t_{0}-\tau_{\max }, t_{0}\right]}\right), \quad \forall t \geq t_{0} \geq 0,
$$

along the trajectory of (4). Furthermore, $\Sigma_{q}$ is asymptotically stable when $\Sigma_{q}$ is stable and $\lim _{t \rightarrow+\infty} x(t)=0$.

For switched time delay systems described by (4), each switching candidate system can be described by (5). Construct the Lyapunov-Razumikhin function

$$
V_{k_{j}}\left(x_{j}, t\right)=x_{j}^{T}(t) P_{k_{j}} x_{j}(t), \quad t \in\left[t_{j}, t_{j+1}\right]
$$

for (5), then we have

$$
\kappa_{k_{j}}\left\|x_{j}(t)\right\|^{2} \leq V_{k_{j}}\left(t, x_{j}\right) \leq \bar{\kappa}_{k_{j}}\left\|x_{j}(t)\right\|^{2}, \forall x_{j} \in \mathbb{R}^{n},
$$

where $\kappa_{k_{j}}:=\sigma_{\min }\left[P_{k_{j}}\right]>0$ denotes the smallest singular value of $P_{k_{j}}$ and $\bar{\kappa}_{k_{j}}:=\sigma_{\max }\left[P_{k_{j}}\right]>0$ the largest singular value of $P_{k_{j}}$.

The first order model transformation [9] of (5) results in

$$
\begin{aligned}
\dot{x}_{j}(t) & =\left(A_{k_{j}}^{c}+\Delta A_{k_{j}}^{c}(\theta)+\bar{A}_{k_{j}}+\Delta \bar{A}(\theta)\right) x_{j}(t) \\
-\left(\bar{A}_{k_{j}}\right. & +\Delta \bar{A}(\theta)) \int_{-\tau_{k_{j}}}^{0}\left[\left(A_{k_{j}}^{c}+\Delta A_{k_{j}}^{c}(\theta+\varphi)\right) x_{j}(t+\varphi)\right. \\
& \left.+\left(\bar{A}_{k_{j}}+\Delta \bar{A}(\theta+\varphi)\right) x\left(t+\varphi-\tau_{k_{j}}\right)\right] d \varphi
\end{aligned}
$$

where the initial condition $\psi_{j}(t)$ is defined as $\psi_{j}(t)=$ $x_{j-1}(t), t \in\left[t_{j}-2 \bar{\tau}_{k_{j}}, t_{j}\right]$ for $j \in \mathbb{Z}^{+}$, and $\psi_{0}(t)$ defined by

$$
\psi_{0}(t)= \begin{cases}\phi(t), & t \in\left[-\tau_{\max }, 0\right] \\ \phi\left(-\tau_{\max }\right), & t \in\left[-2 \tau_{\max },-\tau_{\max }\right)\end{cases}
$$

By using the Lyapunov-Razumikhin function (9), we obtain the time derivative of $V_{k_{j}}\left(t, x_{j}(t)\right)$ along the trajectory of (11)

$$
\dot{V}_{k_{j}}\left(t, x_{j}\right)=x_{j}^{T}(t) H_{k_{j}}(\theta) x_{j}(t)+h_{k_{j}}\left(t, x_{j}\right)
$$

where

$$
\begin{aligned}
H_{k_{j}}(\theta) & =P_{k_{j}}\left(A_{k_{j}}^{c}+\Delta A_{k_{j}}^{c}(\theta)+\bar{A}_{k_{j}}+\Delta \bar{A}(\theta)\right) \\
& +\left(A_{k_{j}}^{c}+\Delta A_{k_{j}}^{c}(\theta)+\bar{A}_{k_{j}}+\Delta \bar{A}(\theta)\right)^{T} P_{k_{j}}
\end{aligned}
$$

and

$$
\begin{aligned}
h_{k_{j}}\left(t, x_{j}\right)= & -\int_{-\tau_{k_{j}}}^{0}\left[2 x_{j}^{T}(t) P_{k_{j}}\left(\bar{A}_{k_{j}}+\Delta \bar{A}(\theta)\right)\right. \\
& \left(\left(A_{k_{j}}^{c}+\Delta A_{k_{j}}^{c}(\theta+\varphi)\right) x_{j}(t+\varphi)\right. \\
+ & \left.\left.\left(\bar{A}_{k_{j}}+\Delta \bar{A}(\theta+\varphi)\right) x\left(t+\varphi-\tau_{k_{j}}\right)\right)\right] d \varphi .
\end{aligned}
$$


Following similar arguments to [2] and assuming existence of a constant $p_{k_{j}}>1$ satisfying $V_{k_{j}}\left(t+\varphi, x_{j}(t+\varphi)\right)<$ $p_{k_{j}} V_{k_{j}}\left(t, x_{j}(t)\right)$ for $\forall \varphi \in\left[-2 \bar{\tau}_{k_{j}}, 0\right]$, we obtain

$$
\dot{V}_{k_{j}}\left(t, x_{j}\right) \leq-x_{j}^{T}(t) S_{k_{j}} x_{j}(t),
$$

with

$$
\begin{aligned}
S_{k_{j}}:= & -\left\{S^{1}+S^{2}+S^{3}+\gamma_{k_{j}} P_{k_{j}} D_{k_{j}} D_{k_{j}}^{T} P_{k_{j}}\right. \\
& +\bar{\gamma}_{k_{j}} P_{k_{j}} \bar{D}_{k_{j}} \bar{D}_{k_{j}}^{T} P_{k_{j}}+\bar{\gamma}_{k_{j}}^{-1} \bar{E}_{k_{j}}^{T} \bar{E}_{k_{j}}+2 \bar{\tau}_{k_{j}} p_{k_{j}} P_{k_{j}} \\
& \left.+\bar{\tau}_{k_{j}} P_{k_{j}}\left(\bar{A}_{k_{j}}\left(Q_{k_{j}}+\bar{Q}_{k_{j}}\right) \bar{A}_{k_{j}}^{T}+\epsilon_{k_{j}} \bar{D}_{k_{j}} \bar{D}_{k_{j}}^{T}\right) P_{k_{j}}\right\}
\end{aligned}
$$

where

$$
\begin{aligned}
S^{1}= & P_{k_{j}}\left(A_{k_{j}}+B_{k_{j}} K_{k_{j}}+\bar{A}_{k_{j}}\right) \\
& +\left(A_{k_{j}}+B_{k_{j}} K_{k_{j}}+\bar{A}_{k_{j}}\right)^{T} P_{k_{j}} \\
S^{2}= & \gamma_{k_{j}}^{-1}\left(E_{k_{j}}+E_{k_{j}}^{B} K_{k_{j}}\right)^{T}\left(E_{k_{j}}+E_{k_{j}}^{B} K_{k_{j}}\right) \\
S^{3}= & \bar{\tau}_{k_{j}} P_{k_{j}} \bar{A}_{k_{j}}\left(Q_{k_{j}}+\bar{Q}_{k_{j}}\right) \bar{E}_{k_{j}}^{T}\left(\epsilon_{k_{j}} I-\bar{E}_{k_{j}}\left(Q_{k_{j}}\right.\right. \\
& \left.\left.+\bar{Q}_{k_{j}}\right) \bar{E}_{k_{j}}^{T}\right)^{-1} \bar{E}_{k_{j}}\left(Q_{k_{j}}+\bar{Q}_{k_{j}}\right) \bar{A}_{k_{j}}^{T} P_{k_{j}}
\end{aligned}
$$

and $\gamma_{k_{j}}>0, \bar{\gamma}_{k_{j}}>0, \epsilon_{k_{j}}>0$ are arbitrary positive scalars, and $Q_{k_{j}}>0, \bar{Q}_{k_{j}}>0$ are chosen such that

$$
\begin{aligned}
\left(( A _ { k _ { j } } ^ { c } + \Delta A _ { k _ { j } } ^ { c } ( \theta + \varphi ) ) ^ { T } Q _ { k _ { j } } ^ { - 1 } \left(\left(A_{k_{j}}^{c}+\Delta A_{k_{j}}^{c}(\theta+\varphi)\right)\right.\right. & \leq P_{k_{j}} \\
\left(\bar{A}_{k_{j}}+\Delta \bar{A}(\theta+\varphi)\right)^{T} \bar{Q}_{k_{j}}^{-1}\left(\bar{A}_{k_{j}}+\Delta \bar{A}(\theta+\varphi)\right) & \leq P_{k_{j}} .
\end{aligned}
$$

Now our goal is to find the matrices and free variables satisfying the above inequalities. For this purpose we use standard techniques from the literature: define $X_{k_{j}}=P_{k_{j}}^{-1}$, then by using Schur complement and Razumikhin theorem, we have the following result, which is a special version of Theorem 3.2 of [2].

Lemma 3.1: The time varying time delay system (5) is robustly stable if there exist $X_{k_{j}}>0, Q_{k_{j}}>0, \bar{Q}_{k_{j}}>0$, $Y_{k_{j}}$, and scalars $\gamma_{k_{j}}>0, \bar{\gamma}_{k_{j}}>0, \epsilon_{k_{j}}>0, \rho_{k_{j}}>0$, $\bar{\rho}_{k_{j}}>0$, such that

$$
\begin{aligned}
& {\left[\begin{array}{ccc}
X_{k_{j}} & X_{k_{j}} A_{k_{j}}^{T}+Y_{k_{j}}^{T} B_{k_{j}}^{T} & X_{k_{j}} E_{k_{j}}^{T}+Y_{k_{j}}^{T}\left(E_{k_{j}}^{B}\right)^{T} \\
\star & Q_{k_{j}}-\rho_{k_{j}} D_{k_{j}} D_{k_{j}}^{T} & 0 \\
\star & \star & \rho_{k_{j}} I
\end{array}\right] \geq 0} \\
& {\left[\begin{array}{ccc}
X_{k_{j}} & X_{k_{j}} \bar{A}_{k_{j}}^{T} & X_{k_{j}} \bar{E}_{k_{j}}^{T} \\
\star & \bar{Q}_{k_{j}}-\bar{\rho}_{k_{j}} \bar{D}_{k_{j}} \bar{D}_{k_{j}}^{T} & 0 \\
\star & \star & \bar{\rho}_{k_{j}} I
\end{array}\right] \geq 0} \\
& {\left[\begin{array}{cccc}
M_{k_{j}} & R_{12} & X_{k_{j}} \bar{E}_{k_{j}}^{T} & R_{14} \\
\star & -\gamma_{k_{j}} I & 0 & 0 \\
\star & \star & -\bar{\gamma}_{k_{j}} I & 0 \\
\star & \star & \star & N_{k_{j}}
\end{array}\right]<0}
\end{aligned}
$$

where

$$
\begin{gathered}
R_{12}:=X_{k_{j}} E_{k_{j}}^{T}+Y_{k_{j}}^{T}\left(E_{k_{j}}^{B}\right)^{T} \\
R_{14}:=\bar{\tau}_{k_{j}} \bar{A}_{k_{j}}\left(Q_{k_{j}}+\bar{Q}_{k_{j}}\right) \bar{E}_{k_{j}}^{T} \\
M_{k_{j}}=\left(A_{k_{j}}+\bar{A}_{k_{j}}\right) X_{k_{j}}+X_{k_{j}}\left(A_{k_{j}}+\bar{A}_{k_{j}}\right)^{T} \\
+\gamma_{k_{j}} D_{k_{j}} D_{k_{j}}^{T}+\bar{\gamma}_{k_{j}} \bar{D}_{k_{j}} \bar{D}_{k_{j}}^{T}+B_{k_{j}} Y_{k_{j}}+Y_{k_{j}}^{T} B_{k_{j}}^{T} \\
+\bar{\tau}_{k_{j}} \epsilon_{k_{j}} \bar{D}_{k_{j}} \bar{D}_{k_{j}}^{T}+\bar{\tau}_{k_{j}} \bar{A}_{k_{j}}\left(Q_{k_{j}}+\bar{Q}_{k_{j}}\right) \bar{A}_{k_{j}}^{T}+2 \bar{\tau}_{k_{j}} p_{k_{j}} X_{k_{j}}, \\
N_{k_{j}}=-\bar{\tau}_{k_{j}}\left(\epsilon_{k_{j}} I-\bar{E}_{k_{j}}\left(Q_{k_{j}}+\bar{Q}_{k_{j}}\right) \bar{E}_{k_{j}}^{T}\right),
\end{gathered}
$$

and $\star$ denotes the transpose of the symmetric term in symmetric matrices. Furthermore, the state feedback controller

$$
K_{k_{j}}=Y_{k_{j}} X_{k_{j}}^{-1}
$$

is robustly stabilizing $\Sigma_{k_{j}}$, (5).

Note that we can select

$$
w_{k_{j}}:=\sigma_{\min }\left[S_{k_{j}}\right]>0
$$

such that

$$
\dot{V}_{k_{j}}\left(t, x_{j}\right)<-w_{k_{j}}\left\|x_{j}\right\|^{2}
$$

Now we are ready to state the main result on stability of the switched LPV time delay system (4). For a given positive constant $\tau_{D}$, the switching signal set based on the dwell time $\tau_{D}$ is denoted by $S\left[\tau_{D}\right]$, where for any switching signal $q(t) \in S\left[\tau_{D}\right]$, the distance between any consecutive discontinuities of $q(t), t_{j+1}-t_{j}, j \in \mathbb{Z}^{+} \cup\{0\}$, is larger than $\tau_{D}$ [10], [22].

Theorem 3.2: Consider switched LPV time delay system (4) with $l$ state feedback controllers designed for $\theta \in \Theta_{i}$, $i \in \mathcal{F}$ as described by (2) and (3), where each controller $K_{k_{j}}, k_{j} \in \mathcal{F}$, is a robustly stabilizing controller derived from Lemma 3.1. Let the dwell time be defined by $\tau_{D}:=T^{*}+2 \tau_{\max }$, where

$$
T^{*}:=\lambda \mu\left\lfloor\frac{\lambda-1}{\bar{p}-1}+1\right\rfloor,
$$

with $\bar{p}:=\min _{k_{j} \in \mathcal{F}}\left\{p_{k_{j}}\right\}>1,\lfloor\cdot\rfloor$ being the floor integer function, and

$$
\lambda:=\max _{k_{j} \in \mathcal{F}} \frac{\bar{\kappa}_{k_{j}}}{\kappa_{k_{j}}}
$$

and

$$
\mu:=\max _{k_{j} \in \mathcal{F}} \frac{\bar{\kappa}_{k_{j}}}{w_{k_{j}}} .
$$

Then system (4) is asymptotically stable for any switching rule $q(t) \in S\left[\tau_{D}\right]$.

Proof. Here we give a sketch of the proof which follows the same arguments made in [30]. First, it can be shown that there exists a constant $0<\alpha<1$, such that

$$
\left|x_{j}\right|_{\left[t_{j}+\bar{T}, t_{j+1}\right]} \leq \alpha \delta_{j}
$$

with $\delta_{0}$ is defined as $\delta_{0}:=|\psi|_{\left[-2 \tau_{\max }, 0\right]}=|\phi|_{\left[-\tau_{\max }, 0\right]}$.

Now recall that $t_{j+1}-t_{j}>\tau_{D}$. Therefore $t_{j+1}-t_{j} \geq \bar{T}+$ $2 \tau_{\max } \geq \bar{T}+2 \bar{\tau}_{k_{j+1}}$. Also notice that $\psi_{j+1}(t)=x_{j}(t), t \in$ $\left[t_{j+1}-2 \bar{\tau}_{k_{j+1}}, t_{j+1}\right]$. We have

$$
\begin{aligned}
& \left|\psi_{j+1}\right|_{\left[t_{j+1}-2 \bar{\tau}_{k_{j+1}}, t_{j+1}\right]}=\left|x_{j}\right|_{\left[t_{j+1}-2 \bar{\tau}_{k_{j+1}}, t_{j+1}\right]} \\
& \leq\left|x_{j}\right|_{\left[t_{j}+\bar{T}, t_{j+1}\right]} \leq \alpha \delta_{j}:=\delta_{j+1} .
\end{aligned}
$$

Therefore we obtain a convergent sequence $\left\{\delta_{i}\right\}, i=$ $0,1,2, \ldots$, where $\delta_{i}=\alpha^{i} \delta_{0}$.

Meanwhile, Proposition 3.2 of [30] implies

$\left|x_{j}\right|_{\left[t-2 \bar{\tau}_{k_{j}}, t\right]} \leq \sqrt{\frac{\bar{\kappa}_{k_{j}}}{\kappa_{k_{j}}}}\left|x_{j}\right|_{\left[t_{j}-2 \bar{\tau}_{k_{j}}, t_{j}\right]}, \quad \forall t \in\left[t_{j}, t_{j+1}\right]$. 
Hence

$$
\begin{aligned}
& \sup _{t \in\left[t_{j}, t_{j+1}\right]}\left\|x_{j}(t)\right\| \\
\leq & \sup _{t \in\left[t_{j}, t_{j+1}\right]}\left|x_{j}\right|_{\left[t-2 \bar{\tau}_{k_{j}}, t\right]} \leq \sqrt{\lambda}\left|x_{j}\right|_{\left[t_{j}-2 \bar{\tau}_{k_{j}}, t_{j}\right]} \\
\leq & \sqrt{\lambda} \delta_{j}=\alpha^{j} \sqrt{\lambda} \delta_{0}
\end{aligned}
$$

which implies the asymptotic stability of the switched time delay system $\Sigma_{q}$, (4), with the switching signal $q(t) \in S_{\left[\tau_{D}\right]}$.

As depicted in Figure 2, two possible switching schemes [29] are (a) critical-point switching, (b) hysteresis switching. For the critical-point switching, the stability of the closedloop system cannot be guaranteed. In fact, in the worst case where $\theta(t)$ oscillates within a neighborhood of $c_{i, i+1}$, fast switching or chattering will happen, which may violate the dwell time requirement. The following corollary provides a sufficient condition for the hysteresis switching scheme over robustly stabilizing controller set $\left\{K_{1}, \ldots, K_{l}\right\}$.

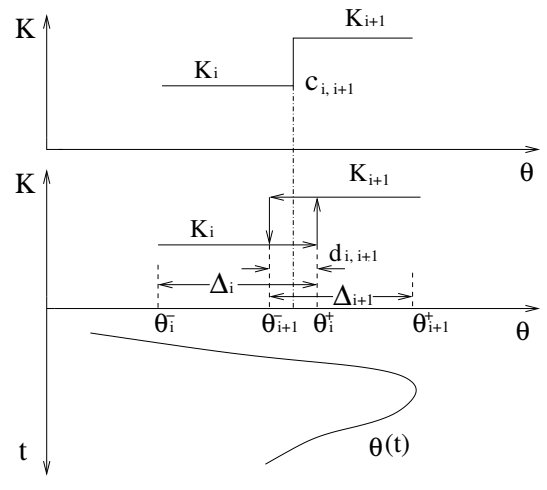

Fig. 2. Switching logic

Corollary 3.3: Consider the switched system $\Sigma_{q}$, (4), with hysteresis switching over the controller set $\left\{K_{1}, \ldots, K_{l}\right\}$. Assume that the operating range $\Theta_{i}$ obeys (3) and the controllers $K_{i}$ are designed according to Lemma 3.1. Then, a sufficient condition for asymptotic stability of (4) is

$$
|\dot{\theta}(t)|<\min _{i \in \mathcal{F}}\left\{\frac{\left|d_{i, i+1}\right|}{\tau_{D}}\right\}
$$

where $d_{i, i+1}=\Theta_{i} \cap \Theta_{i+1}$ is the $i^{t h}$ hysteresis interval as shown in Figure 2 (b) and $\tau_{D}$ is the dwell time given in Theorem 3.2.

Proof. For simplicity, we consider only two neighboring controllers, i.e. $K_{i}$ and $K_{i+1}$ in switching time interval $\left[t_{j}, t_{j+1}\right), j \in \mathbb{Z}^{+} \cup\{0\}$. As discussed in Theorem 3.2, $t_{j+1}-t_{j}>\tau_{D}$ should be satisfied to guarantee stability of the switching system, which requires the currently working controller $K_{i}$ to hold on for an amount of time time at least $\tau_{D}$. In the worst case of switching where $\theta(t)$ oscillates around the center of the interval $d_{i, i+1}$, with amplitude $\left|d_{i, i+1}\right| / 2$, the condition $|\dot{\theta}(t)|<d_{i, i+1} / \tau_{D}$ is sufficient to guarantee stability of the switched system.

\section{NUMERICAL EXAMPLE}

In this section we consider an LPV system which cannot be stabilized by a single controller using the technique of [2]. By separating the region of operation into two overlapping intervals and designing two controllers (one for each interval) as proposed in [2], and using hysteresis switching between these two controllers, as proposed in Corollary 3.3 , it is possible to stabilize the overall system for the whole region of operation.

Let the parameters of (1) be given as

$$
\begin{gathered}
A(\theta)=\left[\begin{array}{rr}
-2.5-1 \theta & -0.75-0.5 \theta \\
-1 & -1.95+0.1 \theta
\end{array}\right] \\
\bar{A}(\theta)=\left[\begin{array}{rr}
-1 & 0 \\
-0.2-0.5 \theta & -1
\end{array}\right] \quad B(\theta)=\left[\begin{array}{ll}
1 & 1
\end{array}\right]^{T}
\end{gathered}
$$

$\tau(\theta)=0.15-0.05 \theta$ and $\theta(t)=\cos \left(\omega_{o} t\right)$. Clearly if $\omega_{o}$ is too large then (28) is not satisfied. We will discuss switched controller design for this system and try to determine how large $\omega_{o}$ can get. In order to answer this question, first robust stability regions must be determined in the parameter space, and then a dwell time must be computed.

Note that $\theta \in[-1,1]=\Theta$. Let $\theta=0$ in the above matrices to define the nominal values of $A, \bar{A}$ and $\bar{\tau}=\max _{\theta \in \Theta}=0.2$. Further define

$$
E=\left[\begin{array}{ll}
1 & 0.5 \\
0 & 0.1
\end{array}\right] \quad \bar{E}=\left[\begin{array}{rr}
0 & 0 \\
0.5 & 0
\end{array}\right], \quad E_{B}=\left[\begin{array}{l}
0 \\
0
\end{array}\right],
$$

and $D=\bar{D}=I$, to cover the matrices in the whole parameter space. With these parameters, Robust Control Toolbox of Matlab cannot find a feasible solution to the LMIs of [2], summarized in Lemma 3.1. This means that a single state feedback controller cannot be found using this approach, for the whole range of $\theta \in[-1,1]$.

In the light of this observation define $\theta_{1}=0.5, \theta_{2}=-0.5$ and two parameter intervals

$$
\Theta_{1}=[-0.1,1] \quad \Theta_{2}=[-1,0.1]
$$

for which two separate controllers $K_{1}$ and $K_{2}$ are to be designed and switched according to the hysteresis curve depicted in Figure 2. For this purpose we define two nominal systems and uncertainty bounds and try to find solutions to the LMIs of Lemma 3.1:

$$
\begin{array}{cc}
A_{1}=\left[\begin{array}{rr}
-3 & -1 \\
-1 & -1.9
\end{array}\right] \quad \bar{A}_{1}=\left[\begin{array}{rr}
-1 & 0 \\
-0.45 & -1
\end{array}\right] \\
A_{2}=\left[\begin{array}{rr}
-2 & -0.5 \\
-1 & -2
\end{array}\right] \quad \bar{A}_{2}=\left[\begin{array}{rr}
-1 & 0 \\
0.05 & -1
\end{array}\right] \\
E_{1}=E_{2}=\left[\begin{array}{rr}
0.6 & 0.3 \\
0 & 0.06
\end{array}\right] \quad \bar{E}_{1}=\bar{E}_{2}=\left[\begin{array}{rr}
0 & 0 \\
0.3 & 0
\end{array}\right]
\end{array}
$$

$D_{1}=D_{2}=\bar{D}_{1}=\bar{D}_{2}=I$, and $\bar{\tau}_{1}=\max _{\theta \in \Theta_{1}}=0.155$ sec., $\bar{\tau}_{2}=\max _{\theta \in \Theta_{2}}=0.20 \mathrm{sec}$. For these systems Robust Control Toolbox of Matlab can solve the LMIs with the free parameters $p_{1}=p_{2}=2.9$, and the resulting controllers

$$
K_{1}=\left[\begin{array}{ll}
1.201 & 0.816
\end{array}\right] \quad K_{2}=\left[\begin{array}{ll}
0.147 & 0.407
\end{array}\right]
$$


gives a dwell time $\tau_{D}=0.83 \mathrm{sec}$. For this example the size of the intersection $\Theta_{1} \cap \Theta_{2}$ is $\left|d_{1,2}\right|=0.2$. Therefore, we can guarantee stability for

$$
|\dot{\theta}|<0.2 / 0.83 \approx 0.24
$$

This means that we can allow $\omega_{o}=\sup _{t \geq 0}|\dot{\theta}(t)|$ to be in the interval $\omega_{o} \in(0,0.24)$. In order to enlarge this range of allowable $\omega_{o}$ we can try increasing $\left|d_{1,2}\right|$, which requires higher values for the entries of $E_{i}$ and $\bar{E}_{i}, i=1,2$. On the other hand, increasing the entries of these matrices lead to higher $\tau_{D}$ which in return decreases the size of $|\dot{\theta}|$. With all the other parameters fixed we were able to increase the entries of $E_{i}$ 's and $\bar{E}_{i}$ 's by a factor $(1+\delta)$ with $\delta=0.1$, that leads to $\left|d_{1,2}\right|=0.32$, with the corresponding $\tau_{D}=$ $0.98 \mathrm{sec}$., so the largest allowable $\omega_{0}$ can be increased to $0.32 / 0.98=0.33 \mathrm{rad} / \mathrm{sec}$. The table given below illustrates the effect of $\delta$ on the ratio $\left|d_{1,2}\right| / \tau_{D}$.

\begin{tabular}{|c||c|c|c|c|c|c|}
\hline$\delta$ & 0 & 0.03 & 0.05 & 0.10 & 0.12 & 0.15 \\
\hline \hline$p_{1}=p_{2}$ & 2.9 & 2.67 & 2.44 & 1.86 & 1.70 & 1.60 \\
\hline$\tau_{D}$ & 0.83 & 0.89 & 0.91 & 0.98 & 1.20 & 1.61 \\
\hline$\left|d_{1,2}\right| / \tau_{D}$ & 0.24 & 0.27 & 0.28 & 0.33 & 0.29 & 0.24 \\
\hline
\end{tabular}

We should also mention that the dwell time computation depends heavily on the selection of the free parameters $p_{i}$ 's; unfortunately, it is not easy to determine the best $p_{i}$ 's minimizing $\tau_{D}$. In the computations for above table we took $p_{1}=p_{2}$ and searched for the minimum dwell time. However, a smaller $\tau_{D}$ might be possible to obtain by doing a bruteforce search over the two dimensional space of $\left(p_{1}, p_{2}\right)$.

\section{Conclusions}

By an extension of [30], a dwell time based hysteresis switching control mechanism is proposed for stabilization of parameter varying time delay systems. The results of [2] are used to compute memoryless state feedback controllers so that robust stability is achieved for intersecting operating intervals which cover the whole parameter space. The approach is illustrated with a numerical example.

Since the approach of [30] is valid for stability of systems under arbitrary switching, there is some conservatism in our main result; because, hysteresis switching mechanism is not an arbitrary switching when we have three or more candidate systems. Possible future studies include conservatism analysis in this approach. Also, output feedback design, and delay in the feedback loop versions of the same problem are open for future studies.

\section{REFERENCES}

[1] P. Apkarian, P. Gahinet and G. Becker, "Self-scheduled $\mathcal{H}^{\infty}$ control of linear parameter-varying systems: a design example", Automatica, vol. 31, pp. 1251-1261, 1995.

[2] Y.-Y. Cao, Y.-X. Sun, and C. Cheng, "Delay-dependent robust stabilization of uncertain systems with multiple state delays", IEEE Trans. Automatic Control, 43:1608-1612, 1998.

[3] C. De Persis, R. De Santis, and S. Morse, "Supervisory control with state-dependent dwell-time logic and constraints," Automatica, 40:269-275, 2004.

[4] L. Dugard and E.I. Verriest, Eds., Stability and Control of Time-Delay Systems, Springer, London, New York, 1998.
[5] K. Gu and S.-I. Niculescu, "Survey on recent results in the stability and control of time-delay systems," ASME Journal of Dynamic Systems, Measurement, and Control, 125:158-165, 2003.

[6] J. C. Geromel and P. Colaneri, "Stability and stabilization of continouous-time switched linear systems," SIAM J. Control and Optimization, vol. 45 (2006), pp. 1915-1930.

[7] J. C. Geromel, P. Colaneri and P. Bolzern, "Dynamic output feedback control of switched linear systems," IEEE Trans. Automatic Control, vol. 53 (2008), pp. 720-733.

[8] K. Gu, V.L. Kharitonov, and J. Chen, Stability and Robust Stability of Time-Delay Systems, Birkhauser, Boston, 2003.

[9] J. Hale and S. Verduyn Lunel, Introduction to Functional Differential Equations, Springer-Verlag, New York, 1993.

[10] J. Hespanha and S. Morse, "Stability of switched systems with average dwell-time", Proc. of the 38th Conf. on Decision and Contr., Phoenix AZ, December 1999, pp. 2655-2660.

[11] J. Hespanha, "Uniform stability of switched linear systems: extension of Lasalle's invariance principle," IEEE Trans. Automatic Control, 49:470-482, 2004.

[12] J. Hespanha, D. Liberzon, and S. Morse, "Overcoming the limitations of adaptive control by means of logic-based switching," System \& Control Letters, 49:49-65, 2003.

[13] J. Hochcerman-Frommer, S. Kulkarni, and P. Ramadge, "Controller switching based on output prediction errors," IEEE Trans. Automatic Control, 43:596-607, 1998.

[14] F. Kelly, "Mathematical modelling of the Internet," in Mathematics Unlimited - 2001 and Beyond, B. Engquist and W. Schmid, Eds., Springer-Verlag, Berlin, 2001, pp. 685-702.

[15] V.L. Kharitonov, "Robust stability analysis of time delay systems: a survey," Annual Reviews in Control, 23:185-196, 1999.

[16] V.L. Kharitonov and D. Hinrichsen, "Exponential estimates for time delay systems," System \& Control Letters, 53(5):395-405, 2004.

[17] V. Kulkarni, M. Jun, and J. Hespanha, "Piecewise quadratic Lyapunov functions for piecewise affine time delay systems," in Proc. of the American Control Conference, Boston, MA, June-July 2004, pp. 3885 3889.

[18] J.-W. Lee, "On uniform stabilization of discrete-time linear parametervarying control systems", IEEE Trans. Automatic Control, vol. 51, pp. 1714-1721, 2006

[19] D. Liberzon and S. Morse, "Basic problems in stability and design of switched systems," IEEE Control Systems Magazine, 19:59-70, 1999.

[20] B. Lu and F. Wu, "Switching LPV control designs using multiple parameter-dependent Lyapunov fucntions", Automatica, vol. 40, pp. 1973-1980, 2004

[21] V. Montagner, V. Leite, S. Tarbouriech, and P. Peres, "Stability and stabilizability of discrete-time switched linear systems with state delay," in Proc. of the American Control Conference, 2005, Portland OR, June 2005, pp. 3806-3811.

[22] S. Morse, "Supervisory control of families of linear set-point controllers: part 1: exact matching," IEEE Trans. Automatic Control, 41:1413-1431, 1996.

[23] S.-I. Niculescu, Delay Effects on Stability: A Robust Control Approach, Lecture Notes in Control and Information Sciences No. 269, SpringerVerlag, Heidelberg, 2001

[24] W. Rugh and J. Shamma, "Research on gain scheduling", Automatica, vol. 36, pp. 1401-1425, 2000.

[25] A. Savkin, E. Sakfidas and R. Evans, "Robust output feedback stabilizability via controller switching", System and Control Letters, vol. 29, pp. 81-90, 1996.

[26] E. Skafidas, R. Evans, A. Savkin, and I. Peterson, "Stability results for switched controller systems," Automatica, 35:553-564, 1999.

[27] F. Wu and K. Grigoriadis, "LPV systems with parameter-varying time delays: analysis and control," Automatica, 37:221-229, 2001.

[28] P. Yan and H. Özbay, "Robust controller design for AQM and $\mathcal{H}^{\infty}$ performance analysis," in Advances in Communication Control Networks, S. Tarbouriech, C. Abdallah, and J. Chiasson, Eds., Lecture Notes in Control and Information Sciences, No. 308, Springer-Verlag, New York, 2005, pp. 49-64.

[29] P. Yan and H. Özbay, "On switching $\mathcal{H}^{\infty}$ controllers for a class of linear parameter varying systems," System \& Control Letters, 56(78):504-511, 2007.

[30] P. Yan and H. Özbay, "Stability analysis of switched time delay systems," SIAM Journal on Control and Optimization, 47(2):936-949, 2008. 